\title{
Multiple Criteria Decision Making Based on Discrete Linguistic Stochastic Variables
}

\author{
Jian Ren, ${ }^{1,2}$ Yang Gao, ${ }^{3}$ and Can Bian ${ }^{4}$ \\ ${ }^{1}$ School of Computer and Information Engineering, Hunan University of Commerce, Changsha 410205, China \\ ${ }^{2}$ School of Business Administration, Hunan University, Changsha 410082, China \\ ${ }^{3}$ Business School, Central South University, Changsha 410083, China \\ ${ }^{4}$ Academic Affairs Office, Hunan Normal University, Changsha 410081, China \\ Correspondence should be addressed to Jian Ren; renwoxing0705@126.com
}

Received 3 December 2012; Revised 4 February 2013; Accepted 4 February 2013

Academic Editor: Wei-Chiang Hong

Copyright (c) 2013 Jian Ren et al. This is an open access article distributed under the Creative Commons Attribution License, which permits unrestricted use, distribution, and reproduction in any medium, provided the original work is properly cited.

\begin{abstract}
For solving the discrete linguistic stochastic multiple criteria decision making problems with incomplete information, a new decision making method based on the differences between the superiorities and the inferiorities is proposed. According to the two basic parameters which are the possible outcome and the state probability, the superior decision matrix and the inferior decision matrix of the alternative set under each criterion are first worked out. Then, by the differences between the elements on the appropriate locations of these matrices, the corresponding dominant decision matrices are formed. Subsequently, with the help of the weight vector of the criterion set, the weighted integrated dominant decision matrix of the alternative set is built. Consequently, the weighted integrated dominant indices' sum of each alternative is calculated. Thus, the rank of the alternatives comes out. Finally, a numerical example is given. The result shows the superiority of the method.
\end{abstract}

\section{Introduction}

Due to the complexity of the things, the uncertainty of the environment, and the limitation of the ability, people usually have difficulty in describing the cognitive objects in the precise numerical values. In this case, the natural languages with which people are familiar are often used to express their mastery of the cognitive objects. In order to make the natural languages more formal, more accurate, and more operational, some scholars made the reasonable effort and achieved some key results. Zadeh [1-3] defined a linguistic variable. Herrera and Martinez $[4,5]$ proposed a 2-tuple fuzzy linguistic representation model. Later on, some new models were presented for the 2-tuple fuzzy linguistic information [6-9]. Martínez and Herrera [10] overviewed the 2-tuple linguistic representation model, including its extensions, specific methodologies, and applications. They also discussed its challenges. Herrera et al. [11] discussed the multigranularity linguistic term sets because of multiple different information sources. $\mathrm{Xu}$ [12] presented the virtual linguistic term sets.
By the present, the linguistic evaluation information has been widely used in the approximate reasoning [1-3], the group decision making (GDM) [13-17], the multiple criteria decision making (MCDM) [18-22], and the other fields. Herrera et al. [11] considered that the solution scheme of the linguistic multiple criteria decision making (LMCDM) problem should consist of the choices of the linguistic term set with its semantic, the aggregation operator of linguistic information, and the best alternatives. Chiclana et al. [23] presented the main computational models for linguistic aggregation in decision making, including linguistic computational model based on membership functions, linguistic computational model based on type-2 fuzzy sets, linguistic symbolic computational models based on ordinal scales, and 2-tuple linguistic computational model. These models have their own characteristics and are suitable for the different environments or needs. Furthermore, they presented some current trends of the linguistic computational methodology in decision making and identified nine current trends. Mendel [24] traced the historical origins of perceptual computing and 
proposed some testable guidelines for perceptual computing. Mendel and $\mathrm{Wu}$ [25] utilized the linguistic computational methodology to design an interactive perceptual computer to aid people in making subjective judgments.

In some practical uncertain MCDM problems, the criterion values could be expressed by stochastic variables. Therefore, the stochastic multiple criteria decision making (SMCDM) is a valuable research topic. Mareschal [26] considered the uncertainty of the data and explicitly put forward the concept of SMCDM. Some methods were proposed to solve the SMCDM problems from different perspectives, including stochastic dominance (SD), stochastic multicriteria acceptability analysis (SMAA), rough sets (RS), and pairwise comparison. Zaras and Martel [27] applied the $\mathrm{SD}$ to the SMCDM problems. Nowak [28] introduced the preference threshold and vetoed the threshold to improve the above research. Nowak [29, 30] proposed an interactive approach to solve the discrete SMCDM problem with certain probability distribution. Zhang et al. [31] combined the SD and PROMETHEE II to deal with the SMCDM problem and discussed the changing criteria weights' impact on the ranking of the alternatives. Lahdelma et al. [32] proposed the SMAA method to solve the SMCDM problems with imprecise or uncertain probability distributions. In practice, the SMAA method showed the superior performance and was transformed into some variants such as SMAA-2 [33, 34], SMAA-O [35, 36], SMAA-3 [37], SMAA-D [38], SMAA$P$ [39], and SMAA-TRI [40]. Zaras considered combining the RS and SD to solve the MCDM problems. Zaras [41] proposed the multiattribute stochastic dominance for a reduced-number-of-attributes (MSDR) method to solve the SMCDM problems with determine, or stochastic criterion values. Subsequently, Zaras [42] proposed the mixeddata multiattribute dominance for a reduced-number-ofattributes (MMDR) method to solve the SMCDM problems with determined stochastic or fuzzy criterion values. In the above two methods, all operations were concentrated in the pairwise comparison table (PCT). Fan et al. [43] proposed a pairwise comparison method for solving SMCDM problems where the criterion values were stochastic variables with known probability density functions or probability mass functions. In the method, the probabilities on all possible results of pairwise comparisons were partitioned into superior, indifferent, and inferior probabilities.

The SMCDM in a fuzzy environment is called fuzzy stochastic multiple criteria decision making (FSMCDM). It is an important research branch of the uncertain MCDM, and it is also a kind of the common problems in the unpredictable and complex society. In brief, what it solves are the problems of selecting, ranking, or classifying the alternatives whose evaluations are fuzzy stochastic variables on multiple criteria. Zarghami et al. [44] proposed the fuzzy-stochastic ordered weighted averaging (FSOWA) method to solve the FSMCDM problem and applied it to the water resources management. Liu et al. [45] constructed the FSMCDM model based on the interval numbers with normal distributions. Wang and Gong [46] proposed a method based on expectation and hybrid entropy to solve the FSMCDM problems in which the criterion weights were precisely known and the criterion values were interval probability fuzzy stochastic variables. Ren and Gao [47] proposed a method based on the information integration to solve the discrete FSMCDM problems with the states' probabilities and the criterions' weights, and both evaluated the interval numbers and some of the alternatives' evaluations on the criterions missing. In the actual decision making, due to the complexity of the decision making environment and the uncertainty of the decision maker's subjective perception, it is general that the evaluations of the alternatives are linguistic stochastic variables and the information of the decision making factors, such as the weights, the state probabilities, the risk attitude and the preference given by the decision maker, may be uncertain or incomplete. At present, there's little concern on the linguistic stochastic multiple criteria decision making (LSMCDM) problems. Wang and Feng [48] considered the fuzziness and the randomness of the criterion values represented by the linguistic evaluation terms and attempted to use a cloud model to solve the multiple criteria and group decision making problems. But they did not analyze a numerical example to verify the validity and the feasibility of the method. $\mathrm{Hu}$ et al. [49] used the prospect theory to construct the LSMCDM model. However, they didn't consider the criterion weights with incomplete information. Ren [50] proposed a method based on the cloud model with regard to the LSMCDM problems with incomplete information. Therefore, the research on the SMCDM problems with incomplete information has a great academic value. That the research result is applied to the engineering management activity to assist the relevant manager to make a decision could optimize the decision making process, reduce the decision making risk, and improve the decision making result. So it has an important practical significance.

The possible outcome and the state probability are two basic parameters by which the stochastic variables can be effectively handled. If the comparing two stochastic variables, the parameters have been often considered synthetically. For example, the expected value, the variance, the probability distribution function, and the probability density function derive from the consideration. Based on this idea, the paper discusses a discrete LSMCDM problem with state probabilities and criterion weights represented by interval numbers. In the proposed method, the comparison of the possible outcomes and the differences of the state probabilities between each alternative and the other ones is considered under each criterion. To do so, the rest of this paper is organized as follows. Section 2 describes a generic discrete LSMCDM problem with incomplete information represented by interval numbers. Section 3 presents a method to verify the consistency of incomplete information represented by interval numbers. Section 4 introduces some basic notations and a comparison method of the discrete linguistic stochastic variables. Section 5 defines some special decision matrices to solve the previous MCDM problem. Section 6 gives out the key steps of the decision making method based on the differences between the superiorities and the inferiorities. Section 7 gives an illustrative example. Finally, the discussion about the proposed method is conducted in the last section. 
TABLE 1: The probability distribution of $\xi$.

\begin{tabular}{lcclc}
\hline$\theta_{k}$ & $\theta_{1}$ & $\theta_{2}$ & $\cdots$ & $\theta_{l}$ \\
\hline$p\left(\theta_{k}\right)$ & $p\left(\theta_{1}\right)$ & $p\left(\theta_{2}\right)$ & $\ldots$ & $p\left(\theta_{l}\right)$ \\
$\xi\left(\theta_{k}\right)$ & $\widetilde{A}_{1}$ & $\widetilde{A}_{2}$ & $\ldots$ & $\widetilde{A}_{l}$ \\
\hline
\end{tabular}

\section{Problem Description}

A MCDM problem is defined on a set of the alternatives from which a decision maker has to select the optimal alternative according to some criteria. Suppose there exists an alternative set denoted by $A=\left\{a_{1}, a_{2}, \ldots, a_{m}\right\}$ which consists of $m$ alternatives, the decision maker will select the optimal one from $A$ according to a criterion set denoted by $C=$ $\left\{C_{1}, C_{2}, \ldots, C_{n}\right\}$ which includes $n$ criteria. For convenience, an interval weight vector of the criteria is denoted by $\Omega=$ $\left(\bar{\omega}_{1}, \bar{\omega}_{1}, \ldots, \bar{\omega}_{n}\right)$, where $\bar{\omega}_{j}=\left[\omega_{j}^{L}, \omega_{j}^{R}\right](j \in N=\{1,2, \ldots, n\})$ and $0 \leq \omega_{j}^{L} \leq \omega_{j}^{R} \leq 1$. It represents the relative importance of each criterion. A consistent state space is expressed by $\Theta=\left\{\theta_{1}, \theta_{2}, \ldots, \theta_{l}\right\}$. An interval probability vector of the states is denoted by $\Xi=\left(\bar{p}\left(\theta_{1}\right), \bar{p}\left(\theta_{2}\right), \ldots, \bar{p}\left(\theta_{l}\right)\right)$, where $\bar{p}\left(\theta_{k}\right)=$ $\left[p_{k}^{L}, p_{k}^{R}\right](k \in L=\{1,2, \ldots, l\})$, and $0 \leq p_{k}^{L} \leq p_{k}^{R} \leq$ 1. It represents the probability range of each state. A fuzzy stochastic decision matrix is symbolized by FSDM $=\left(x_{i j}\right)_{m \times n}$, where $x_{i j}(i \in M=\{1,2, \ldots, m\}, j \in N)$ is a discrete linguistic stochastic variable. $x_{i j k}(i \in M, j \in N, k \in L)$ means a linguistic evaluation term of $x_{i j}$ under $\theta_{k}$. That is to say, it is a performance of $a_{i}$ under $C_{j}$ and $\theta_{k}$.

\section{Consistency Verification of Incomplete Information Represented by Interval Numbers}

Clearly, it would be desirable to maintain the consistency level of incomplete information provided by the decision maker. Herrera-Viedma et al. [51] provided the additive consistency property to measure the level of consistency of the incomplete fuzzy preference information provided by the experts in the GDM problems.

Here, a method is proposed to ensure the consistency level of incomplete information represented by the interval numbers provided by the decision maker.

Wang [52] presented a method to verify the interval probability vector of the states. Similarly, according to the principles of the above method, the following definitions and propositions are gotten.

Definition 1. Let $\Omega=\left(\bar{\omega}_{1}, \bar{\omega}_{1}, \ldots, \bar{\omega}_{n}\right)$ be an interval weight vector of the criteria, where $\bar{\omega}_{j}=\left[\omega_{j}^{L}, \omega_{j}^{R}\right](j \in N)$. If $\sum_{j=1}^{n} \omega_{j}^{L} \leq 1$ and $\sum_{j=1}^{n} \omega_{j}^{R} \geq 1, \Omega$ is rational.

If $\Omega$ is not rational, $\Omega$ should be adjusted according to the existing decision making information.

Definition 2. Let $\Omega=\left(\bar{\omega}_{1}, \bar{\omega}_{1}, \ldots, \bar{\omega}_{n}\right)$ be an interval weight vector of the criteria, where $\bar{\omega}_{j}=\left[\omega_{j}^{L}, \omega_{j}^{R}\right](j \in N)$. If for all $\omega_{j} \in\left[\omega_{j}^{L}, \omega_{j}^{R}\right](j \in N)$, always $\exists\left(\omega_{1}, \omega_{2}, \ldots, \omega_{n}\right)$, where $\sum_{j=1}^{n} \omega_{j}=1$ and $\omega_{j} \in\left[\omega_{j}^{L}, \omega_{j}^{R}\right](j \in N) . \Omega$ is feasible.

Obviously, if $\Omega$ is rational, it is not necessarily feasible. However, if $\Omega$ is feasible, it is undoubtedly rational.

Proposition 3. Let $\Omega=\left(\bar{\omega}_{1}, \bar{\omega}_{1}, \ldots, \bar{\omega}_{n}\right)$ be an interval weight vector of the criteria, where $\bar{\omega}_{j}=\left[\omega_{j}^{L}, \omega_{j}^{R}\right](j \in N)$. If $\Omega$ is rational and unfeasible, $\Omega$ can be adjusted according to the following formulas:

$$
\begin{aligned}
& \widehat{\omega}_{j}^{L}=\max \left(\omega_{j}^{L}, 1-\sum_{\substack{h=1 \\
h \neq j}}^{n} \omega_{h}^{R}\right), \quad j \in N, \\
& \widehat{\omega}_{j}^{R}=\min \left(\omega_{j}^{R}, 1-\sum_{\substack{h=1 \\
h \neq j}}^{n} \omega_{h}^{L}\right), \quad j \in N .
\end{aligned}
$$

We have the following proof procedure:

$$
\begin{aligned}
& \because \widehat{\omega}_{s}^{L} \leq \omega_{s} \leq \widehat{\omega}_{s}^{R}, \text { namely, } \max \left(\omega_{s}^{L}, 1-\sum_{t=1, t \neq s}^{n} \omega_{t}^{R}\right) \leq \\
& \omega_{s} \leq \min \left(\omega_{s}^{R}, 1-\sum_{t=1, t \neq s}^{n} \omega_{t}^{L}\right) \\
& \therefore 1-\sum_{t=1, t \neq s}^{n} \omega_{t}^{R} \leq \omega_{s} \leq 1-\sum_{t=1, t \neq s}^{n} \omega_{t}^{L}, \text { simulta- } \\
& \text { neously, } \omega_{s}^{L} \leq \omega_{s} \leq \omega_{s}^{R} \\
& \because 1 \leq \omega_{s}+\sum_{t=1, t \neq s}^{n} \omega_{t}^{R}, \text { simultaneously, } \omega_{s}+ \\
& \sum_{t=1, t \neq s}^{n} \omega_{t}^{L} \leq 1 \\
& \therefore \exists \omega_{t} \in\left[\omega_{t}^{L}, \omega_{t}^{R}\right](t \in N-\{s\}), \text { and then, } \sum_{t=1, t \neq s}^{n} \omega_{t}+ \\
& \omega_{s}=1 .
\end{aligned}
$$

Proposition 3 has been proved. In the actual decision making, after the rationality of $\Omega$ is verified, regardless of its feasibility, it can be adjusted by formula (1) to ensure its feasibility.

Definition 4. Let $\Omega=\left(\bar{\omega}_{1}, \bar{\omega}_{1}, \ldots, \bar{\omega}_{n}\right)$ be an interval weight vector of the criteria, where $\bar{\omega}_{j}=\left[\omega_{j}^{L}, \omega_{j}^{R}\right](j \in N)$. If $\Omega$ is rational and feasible, it is consistent.

\section{Discrete Linguistic Stochastic Variables}

Definition 5. Let $\xi$ be a function from a probability space $(\Theta, A, \operatorname{Pr})$ to a linguistic variable set. If $\operatorname{pos}\left(\xi\left(\theta_{k}\right) \in B\right)$ is a measurable function for each Borel set $B$ on the real number set, where $\theta_{k} \in \Theta, \xi$ is a linguistic stochastic variable.

Definition 6. If $\xi$ satisfies the relation given in Table 1 , it is a discrete linguistic stochastic variable.

In Table $1, \theta_{k}(k \in L)$ is a state, $p\left(\theta_{k}\right)$ is the probability of $\theta_{k}, \xi\left(\theta_{k}\right)$ is a linguistic variable, and $\widetilde{A}_{k}$ is linguistic evaluation term. Specifically, $p\left(\theta_{k}\right)$ may be certain or uncertain.

In order to compare the independent discrete linguistic stochastic variables effectively, the method based on the differences between the superiorities and the inferiorities is given. 
TABLE 2: The probability distribution of $\xi^{1}$.

\begin{tabular}{lcclc}
\hline$\theta_{k}^{1}$ & $\theta_{1}^{1}$ & $\theta_{2}^{1}$ & $\cdots$ & $\theta_{l_{1}}^{1}$ \\
\hline$p\left(\theta_{k}^{1}\right)$ & $p\left(\theta_{1}^{1}\right)$ & $p\left(\theta_{2}^{1}\right)$ & $\cdots$ & $p\left(\theta_{l_{1}}^{1}\right)$ \\
$\xi\left(\theta_{k}^{1}\right)$ & $\widetilde{A}_{1}^{1}$ & $\widetilde{A}_{2}^{1}$ & $\cdots$ & $\widetilde{A}_{l_{1}}$ \\
\hline
\end{tabular}

TABLE 3: The probability distribution of $\xi^{2}$.

\begin{tabular}{lcclc}
\hline$\theta_{k}^{2}$ & $\theta_{1}^{2}$ & $\theta_{2}^{2}$ & $\cdots$ & $\theta_{l_{2}}^{2}$ \\
\hline$p\left(\theta_{k}^{2}\right)$ & $p\left(\theta_{1}^{2}\right)$ & $p\left(\theta_{2}^{2}\right)$ & $\cdots$ & $p\left(\theta_{l_{2}}^{2}\right)$ \\
$\xi\left(\theta_{k}^{2}\right)$ & $\widetilde{A}_{1}^{2}$ & $\widetilde{A}_{2}^{2}$ & $\cdots$ & $\widetilde{A}_{l_{2}}$ \\
\hline
\end{tabular}

Definition 7. $\xi^{1}$ and $\xi^{2}$ are two mutually independent discrete linguistic stochastic variables which satisfy

$$
p\left(\xi^{1} \xi^{2}\right)=p\left(\xi^{1}\right) p\left(\xi^{2}\right)
$$

Let $\xi^{1}$ and $\xi^{2}$ be two mutually independent discrete linguistic stochastic variables. And the probability distributions of $\xi^{1}$ and $\xi^{2}$ are shown in Tables 2 and 3 .

Definition 8. The superior index of $\xi^{1}$ and $\xi^{2}$ is defined as follows:

$$
\operatorname{SI}\left(\xi^{1}, \xi^{2}\right)=\sum_{\widetilde{A}_{k_{1}}^{1}>\widetilde{A}_{k_{2}}^{2}} p\left(\theta_{k_{1}}^{1}\right) p\left(\theta_{k_{2}}^{2}\right), \quad\left(k_{1} \in L_{1}, k_{2} \in L_{2}\right) .
$$

For convenience, $\operatorname{SI}\left(\xi^{1}, \xi^{2}\right)$ is denoted by $\mathrm{SI}^{12}$.

Definition 9. The inferior index of $\xi^{1}$ and $\xi^{2}$ is defined as follows:

$$
\mathrm{II}\left(\xi^{1}, \xi^{2}\right)=\sum_{\widetilde{A}_{k_{1}}^{1}<\widetilde{A}_{k_{2}}^{2}} p\left(\theta_{k_{1}}^{1}\right) p\left(\theta_{k_{2}}^{2}\right), \quad\left(k_{1} \in L_{1}, k_{2} \in L_{2}\right) .
$$

For convenience, $\mathrm{II}\left(\xi^{1}, \xi^{2}\right)$ is denoted by $\mathrm{II}^{12}$.

Definition 10. The equivalent index of $\xi^{1}$ and $\xi^{2}$ is defined as follows:

$$
\mathrm{EI}\left(\xi^{1}, \xi^{2}\right)=\sum_{\widetilde{A}_{k_{1}}^{1}=\widetilde{A}_{k_{2}}^{2}} p\left(\theta_{k_{1}}^{1}\right) p\left(\theta_{k_{2}}^{2}\right), \quad\left(k_{1} \in L_{1}, k_{2} \in L_{2}\right) .
$$

For convenience, $\mathrm{EI}\left(\xi^{1}, \xi^{2}\right)$ is denoted by $\mathrm{EI}^{12}$.

Proposition 11. $\mathrm{SI}^{12}, \mathrm{II}^{12}$, and $\mathrm{EI}^{12}$ of $\xi^{1}$ and $\xi^{2}$ satisfy the following conditions:

(1) $\mathrm{SI}^{12} \in[0,1], \mathrm{II}^{12} \in[0,1], \mathrm{EI}^{12} \in[0,1]$,

(2) $\mathrm{SI}^{12}+\mathrm{II}^{12}+\mathrm{EI}^{12}=1$,

(3) $\mathrm{SI}^{12}=\mathrm{II}^{21}$,
(4) $\mathrm{II}^{12}=\mathrm{SI}^{21}$,

(5) $\mathrm{EI}^{12}=\mathrm{EI}^{21}$.

Obviously, Proposition 11 is established.

Definition 12. The dominant index of $\xi^{1}$ and $\xi^{2}$ is defined as follows:

$$
\mathrm{DI}\left(\xi^{1}, \xi^{2}\right)=\mathrm{SI}^{12}-\mathrm{II}^{12}
$$

For convenience, $\mathrm{DI}\left(\xi^{1}, \xi^{2}\right)$ is denoted by $\mathrm{DI}^{12}$.

Proposition 13. $\mathrm{DI}^{12}$ of $\xi^{1}$ and $\xi^{2}$ satisfies the following conditions:
(1) $\mathrm{DI}^{12} \in[-1,1]$,
(2) $\mathrm{DI}^{12}=-\mathrm{DI}^{21}$.

Obviously, Proposition 13 is established.

Proposition 14. The performances of $\xi^{1}$ and $\xi^{2}$ can be compared by $\mathrm{DI}^{12}$ as follows.

(1) If $\mathrm{DI}^{12}>0, \xi^{1}>\xi^{2}$.

(2) If $\mathrm{DI}^{12}=0, \xi^{1} \sim \xi^{2}$.

(3) If $\mathrm{DI}^{12}<0, \xi^{1} \prec \xi^{2}$.

Obviously, Proposition 14 is established.

If the probabilities of $\xi^{1}$ and $\xi^{2}$ are interval numbers, Propositions 11, 13, and 14 are uncertain to be strictly established.

\section{Decision Matrices of the Discrete Linguistic Stochastic Multiple Criteria Decision Making}

Definition 15. The superior decision matrix under $C_{j}(j \in N)$ is denoted by $\mathrm{SDM}_{j}=\left(\mathrm{SI}_{j}^{i_{1} i_{2}}\right)_{m \times m}\left(i_{1}, i_{2} \in M\right)$, where $\mathrm{SI}_{j}^{i_{1} i_{2}}$ is the superior index of $a_{i_{1}}$ and $a_{i_{2}}$ under $C_{j}$. If $i_{1}=i_{2}, \operatorname{SI}_{j}^{i_{1} i_{2}}=\phi$.

Definition 16. The inferior decision matrix under $C_{j}(j \in N)$ is denoted by $\operatorname{IDM}_{j}=\left(\mathrm{II}_{j}^{i_{1} i_{2}}\right)_{m \times m}\left(i_{1}, i_{2} \in M\right)$, where $\mathrm{II}_{j}^{i_{1} i_{2}}$ is the inferior index of $a_{i_{1}}$ and $a_{i_{2}}$ under $C_{j}$. If $i_{1}=i_{2}, \mathrm{II}_{j}^{i_{1} i_{2}}=\phi$.

Definition 17. The equivalent decision matrix under $C_{j}(j \in$ $N)$ is denoted by $\mathrm{EDM}_{j}=\left(\mathrm{EI}_{j}^{i_{1} i_{2}}\right)_{m \times m}\left(i_{1}, i_{2} \in M\right)$, where $\mathrm{EI}_{j}^{i_{1} i_{2}}$ is the equivalent index of $a_{i_{1}}$ and $a_{i_{2}}$ under $C_{j}$. If $i_{1}=i_{2}$, $\mathrm{EI}_{j}^{i_{1} i_{2}}=\phi$.

Definition 18. The dominant decision matrix under $C_{j}(j \in$ $N)$ is denoted by $\mathrm{DDM}_{j}=\left(\mathrm{DI}_{j}^{i_{1} i_{2}}\right)_{m \times m}\left(i_{1}, i_{2} \in M\right)$, where $\mathrm{DI}_{j}^{i_{1} i_{2}}$ is the dominant index of $a_{i_{1}}$ and $a_{i_{2}}$ under $C_{j}$. If $i_{1}=i_{2}$, $\mathrm{DI}_{j}^{i_{1} i_{2}}=\phi$. 
Definition 19. The weighted integrated dominant decision matrix is denoted by $\mathrm{DDM}_{\omega}=\left(\mathrm{DI}_{\omega}^{i_{1} i_{2}}\right)_{m \times m}\left(i_{1}, i_{2} \in M\right)$, where $\mathrm{DI}_{\omega}^{i_{1} i_{2}}=\sum_{j=1}^{n} \omega_{j} \times \mathrm{DI}_{j}^{i_{1} i_{2}}$ is the weighted integrated dominant index of $a_{i_{1}}$ and $a_{i_{2}}$ under the criterion set. If $i_{1}=i_{2}, \mathrm{DI}_{j}^{i_{1} i_{2}}=\phi$.

\section{Decision Procedure}

To recap, a decision procedure based on the differences between the superiorities and the inferiorities for the discrete LSMCDM problems with incomplete information can be shown as follows.

Step 1. $\mathrm{SDM}_{j}$ and $\mathrm{IDM}_{j}$ under $C_{j}(j \in N)$ are constructed.

Step 2. $\mathrm{DDM}_{j}$ under $C_{j}(j \in N)$ is constructed.

Step 3. $\mathrm{DDM}_{\omega}$ is constructed.

Step 4. The weighted integrated dominant indices' sum of $a_{i_{1}}\left(i_{1} \in M\right)$ denoted by $\mathrm{SDI}^{i_{1}}$ is calculated as follows:

$$
\mathrm{SDI}^{i_{1}}=\sum_{i_{2}=1, i_{2} \neq i_{1}}^{m} \mathrm{DI}_{\omega}^{i_{1} i_{2}}, \quad\left(i_{1} \in M\right)
$$

Step 5. According to $\mathrm{SDI}^{i_{1}}\left(i_{1} \in M\right)$, the ranking order of the alternatives can be determined.

When the state probabilities and the criterion weights are certain evaluations, the above steps are used to make the decision. If not, the uncertain evaluations need to be specially processed.

The discrete LSMCDM problems with state probabilities and criterion weights being interval numbers are considered in the paper. In the problems like these, the state probabilities and the criterion weights are processed by the operation laws of the interval numbers. And the order of the interval numbers is gotten by the possibility degree matrix and the ranking weight vector.

\section{Illustrative Example}

In the section, an example is used to show the decision procedure in Section 5. Let $A=\left\{a_{1}, a_{2}, \ldots, a_{5}\right\}$ be an alternative set, $C=\left\{C_{1}, C_{2}, \ldots, C_{5}\right\}$ be a criterion set, and $\Theta=$ $\left\{\theta_{1}, \theta_{2}, \ldots, \theta_{l}\right\}$ be a consistent state space. The interval weight vector of the criteria is $\Omega=\left(\bar{\omega}_{1}=[0.2000,0.2500], \bar{\omega}_{2}=\right.$ $[0.2500,0.3000], \bar{\omega}_{3}=[0.1000,0.2000], \bar{\omega}_{4}=[0.1500,0.2500]$, and $\left.\bar{\omega}_{5}=[0.2000,0.3000]\right)$. The interval probability vector of the states is $\Xi=\left(\bar{p}\left(\theta_{1}\right)=[0.3000,0.5000], \bar{p}\left(\theta_{2}\right)=\right.$ $[0.4000,0.6000]$, and $\left.\bar{p}\left(\theta_{3}\right)=[0.1000,0.3000]\right)$. The linguistic evaluation term set is LETS $=\left\{s_{0}, s_{1}, s_{2}, s_{3}, s_{4}, s_{5}, s_{6}\right\}$, where $s_{0}$ means "very low," $s_{1}$ means "low," $s_{2}$ means "medium low," $s_{3}$ means "medium," $s_{4}$ means "medium high," $s_{5}$ means "high," and $s_{6}$ means "very high" [11].

In this case, the original decision information is shown in Tables $4,5,6,7,8,9,10,11,12$, and 13 . Let the decision maker select the optimal alternative from $A$.

The decision procedure is shown as follows.
TABLE 4: The state probabilities of the alternatives under $C_{1}$.

\begin{tabular}{cccc}
\hline & $\theta_{1}^{i 1}$ & $\theta_{2}^{i 1}$ & $\theta_{3}^{i 1}$ \\
\hline$a_{1}$ & {$[0.1000,0.3000]$} & {$[0.4000,0.5000]$} & {$[0.3000,0.4000]$} \\
$a_{2}$ & {$[0.4000,0.5000]$} & {$[0.2000,0.3000]$} & {$[0.2000,0.3000]$} \\
$a_{3}$ & {$[0.2000,0.3000]$} & {$[0.3000,0.4000]$} & {$[0.4000,0.5000]$} \\
$a_{4}$ & {$[0.3000,0.4000]$} & {$[0.2000,0.3000]$} & {$[0.3000,0.4000]$} \\
$a_{5}$ & {$[0.5000,0.6000]$} & {$[0.1000,0.2000]$} & {$[0.2000,0.3000]$} \\
\hline
\end{tabular}

TABLE 5: The state probabilities of the alternatives under $C_{2}$.

\begin{tabular}{cccc}
\hline & $\theta_{1}^{i 2}$ & $\theta_{2}^{i 2}$ & $\theta_{3}^{i 2}$ \\
\hline$a_{1}$ & {$[0.4000,0.5000]$} & {$[0.2000,0.3000]$} & {$[0.2000,0.3000]$} \\
$a_{2}$ & {$[0.2000,0.3000]$} & {$[0.3000,0.4000]$} & {$[0.4000,0.5000]$} \\
$a_{3}$ & {$[0.3000,0.4000]$} & {$[0.2000,0.3000]$} & {$[0.3000,0.4000]$} \\
$a_{4}$ & {$[0.5000,0.6000]$} & {$[0.1000,0.2000]$} & {$[0.2000,0.3000]$} \\
$a_{5}$ & {$[0.1000,0.3000]$} & {$[0.4000,0.5000]$} & {$[0.3000,0.4000]$} \\
\hline
\end{tabular}

TABLE 6: The state probabilities of the alternatives under $C_{3}$.

\begin{tabular}{cccc}
\hline & $\theta_{1}^{i 3}$ & $\theta_{2}^{i 3}$ & $\theta_{3}^{i 3}$ \\
\hline$a_{1}$ & {$[0.2000,0.3000]$} & {$[0.3000,0.4000]$} & {$[0.4000,0.5000]$} \\
$a_{2}$ & {$[0.3000,0.4000]$} & {$[0.2000,0.3000]$} & {$[0.3000,0.4000]$} \\
$a_{3}$ & {$[0.5000,0.6000]$} & {$[0.1000,0.2000]$} & {$[0.2000,0.3000]$} \\
$a_{4}$ & {$[0.1000,0.3000]$} & {$[0.4000,0.5000]$} & {$[0.3000,0.4000]$} \\
$a_{5}$ & {$[0.4000,0.5000]$} & {$[0.2000,0.3000]$} & {$[0.2000,0.3000]$} \\
\hline
\end{tabular}

TABLE 7: The state probabilities of the alternatives under $C_{4}$.

\begin{tabular}{cccc}
\hline & $\theta_{1}^{i 4}$ & $\theta_{2}^{i 4}$ & $\theta_{3}^{i 4}$ \\
\hline$a_{1}$ & {$[0.3000,0.4000]$} & {$[0.2000,0.3000]$} & {$[0.3000,0.4000]$} \\
$a_{2}$ & {$[0.5000,0.6000]$} & {$[0.1000,0.2000]$} & {$[0.2000,0.3000]$} \\
$a_{3}$ & {$[0.1000,0.3000]$} & {$[0.4000,0.5000]$} & {$[0.3000,0.4000]$} \\
$a_{4}$ & {$[0.4000,0.5000]$} & {$[0.2000,0.3000]$} & {$[0.2000,0.3000]$} \\
$a_{5}$ & {$[0.2000,0.3000]$} & {$[0.3000,0.4000]$} & {$[0.4000,0.5000]$} \\
\hline
\end{tabular}

TABLE 8: The state probabilities of the alternatives under $C_{5}$.

\begin{tabular}{cccc}
\hline & $\theta_{1}^{i 5}$ & $\theta_{2}^{i 5}$ & $\theta_{3}^{i 5}$ \\
\hline$a_{1}$ & {$[0.5000,0.6000]$} & {$[0.1000,0.2000]$} & {$[0.2000,0.3000]$} \\
$a_{2}$ & {$[0.1000,0.3000]$} & {$[0.4000,0.5000]$} & {$[0.3000,0.4000]$} \\
$a_{3}$ & {$[0.4000,0.5000]$} & {$[0.2000,0.3000]$} & {$[0.2000,0.3000]$} \\
$a_{4}$ & {$[0.2000,0.3000]$} & {$[0.3000,0.4000]$} & {$[0.4000,0.5000]$} \\
$a_{5}$ & {$[0.3000,0.4000]$} & {$[0.2000,0.3000]$} & {$[0.3000,0.4000]$} \\
\hline
\end{tabular}

TABLE 9: The discrete linguistic stochastic variable of the alternatives under $C_{1}$.

\begin{tabular}{cccccccccccccccc}
\hline & \multicolumn{1}{c}{$a_{1}$} & & $a_{2}$ & & $a_{3}$ & & $a_{4}$ & & \multicolumn{1}{c}{$a_{5}$} \\
\hline$\theta_{1}^{11}$ & $\theta_{2}^{11}$ & $\theta_{3}^{11}$ & $\theta_{1}^{21}$ & $\theta_{2}^{21}$ & $\theta_{3}^{21}$ & $\theta_{1}^{31}$ & $\theta_{2}^{31}$ & $\theta_{3}^{31}$ & $\theta_{1}^{41}$ & $\theta_{2}^{41}$ & $\theta_{3}^{41}$ & $\theta_{1}^{51}$ & $\theta_{2}^{51}$ & $\theta_{3}^{51}$ \\
M & H & VH & H & M & M & M & M & H & VH & L & M & H & L & P \\
\hline
\end{tabular}

$\mathrm{SDM}_{j}$ and $\mathrm{IDM}_{j}$ under $C_{j}(j \in N=\{1,2, \ldots, 5\})$ are shown in Tables 14, 15, 16, 17, 18, 19, 20, 21, 22, and 23.

$\mathrm{DDM}_{j}$ under $C_{j}(j \in N)$ is shown in Tables $24,25,26,27$, and 28 . 
TABLE 10: The discrete linguistic stochastic variable of the alternatives under $C_{2}$.

\begin{tabular}{ccccccccccccccc}
\hline & \multicolumn{1}{c}{$a_{1}$} & & \multicolumn{1}{c}{$a_{2}$} & & \multicolumn{1}{c}{$a_{3}$} & & $a_{4}$ & & $a_{5}$ \\
\hline$\theta_{1}^{12}$ & $\theta_{2}^{12}$ & $\theta_{3}^{12}$ & $\theta_{1}^{22}$ & $\theta_{2}^{22}$ & $\theta_{3}^{22}$ & $\theta_{1}^{32}$ & $\theta_{2}^{32}$ & $\theta_{3}^{32}$ & $\theta_{1}^{42}$ & $\theta_{2}^{42}$ & $\theta_{3}^{42}$ & $\theta_{1}^{52}$ & $\theta_{2}^{52}$ & $\theta_{3}^{52}$ \\
$\mathrm{M}$ & $\mathrm{H}$ & $\mathrm{VH}$ & $\mathrm{M}$ & $\mathrm{P}$ & $\mathrm{H}$ & $\mathrm{H}$ & $\mathrm{P}$ & $\mathrm{M}$ & $\mathrm{M}$ & $\mathrm{P}$ & $\mathrm{H}$ & $\mathrm{P}$ & $\mathrm{M}$ & $\mathrm{H}$ \\
\hline
\end{tabular}

TABLE 11: The discrete linguistic stochastic variable of the alternatives under $C_{3}$.

\begin{tabular}{lcccccccccccccc}
\hline & \multicolumn{1}{c}{$a_{1}$} & & $a_{2}$ & & $a_{3}$ & & $a_{4}$ & & $a_{5}$ \\
\hline$\theta_{1}^{13}$ & $\theta_{2}^{13}$ & $\theta_{3}^{13}$ & $\theta_{1}^{23}$ & $\theta_{2}^{23}$ & $\theta_{3}^{23}$ & $\theta_{1}^{33}$ & $\theta_{2}^{33}$ & $\theta_{3}^{33}$ & $\theta_{1}^{43}$ & $\theta_{2}^{43}$ & $\theta_{3}^{43}$ & $\theta_{1}^{53}$ & $\theta_{2}^{53}$ & $\theta_{3}^{53}$ \\
M VH & VH & H & M & H & VL & P & VH & M & M & M & L & P & VH \\
\hline
\end{tabular}

TABLE 12: The discrete linguistic stochastic variable of the alternatives under $C_{4}$.

\begin{tabular}{ccccccccccccccc}
\hline & \multicolumn{1}{c}{$a_{1}$} & \multicolumn{1}{c}{$a_{2}$} & \multicolumn{1}{c}{$a_{3}$} & & $a_{4}$ & & \multicolumn{3}{c}{$a_{5}$} \\
\hline$\theta_{1}^{14}$ & $\theta_{2}^{14}$ & $\theta_{3}^{14}$ & $\theta_{1}^{24}$ & $\theta_{2}^{24}$ & $\theta_{3}^{24}$ & $\theta_{1}^{34}$ & $\theta_{2}^{34}$ & $\theta_{3}^{34}$ & $\theta_{1}^{44}$ & $\theta_{2}^{44}$ & $\theta_{3}^{44}$ & $\theta_{1}^{54}$ & $\theta_{2}^{54}$ & $\theta_{3}^{54}$ \\
M & $\mathrm{P}$ & H & VH & H & M & P & VH & H & L & VH & H & VH & M & H \\
\hline
\end{tabular}

TABLE 13: The discrete linguistic stochastic variable of the alternatives under $C_{5}$.

\begin{tabular}{lccccccccccccccc}
\hline & \multicolumn{1}{c}{$a_{1}$} & & $a_{2}$ & & & $a_{3}$ & & $a_{4}$ & & $a_{5}$ \\
\hline$\theta_{1}^{15}$ & $\theta_{2}^{15}$ & $\theta_{3}^{15}$ & $\theta_{1}^{25}$ & $\theta_{2}^{25}$ & $\theta_{3}^{25}$ & $\theta_{1}^{35}$ & $\theta_{2}^{35}$ & $\theta_{3}^{35}$ & $\theta_{1}^{45}$ & $\theta_{2}^{45}$ & $\theta_{3}^{45}$ & $\theta_{1}^{55}$ & $\theta_{2}^{55}$ & $\theta_{3}^{55}$ \\
H & VH & L & VH & H & M & H & VL & VH & H & H & L & M & M & M \\
\hline
\end{tabular}

$\mathrm{DDM}_{\omega}$ is shown in Table 29.

$\operatorname{SDI}^{i_{1}}\left(i_{1} \in M=\{1,2, \ldots, 5\}\right)$ is shown in Table 30 .

The possibility degree matrix denoted by PDM is computed as follows:

PDM

$$
=\left(\begin{array}{lllll}
0.5000 & 0.5185 & 0.5725 & 0.8445 & 0.6840 \\
0.4815 & 0.5000 & 0.5544 & 0.8287 & 0.6673 \\
0.4275 & 0.4456 & 0.5000 & 0.7747 & 0.6144 \\
0.1555 & 0.1713 & 0.2253 & 0.5000 & 0.3462 \\
0.3160 & 0.3327 & 0.3856 & 0.6538 & 0.5000
\end{array}\right) .
$$

The ranking weight vector is calculated as follows:

$$
\mathrm{RV}=(0.2310,0.2266,0.2131,0.1449,0.1844) .
$$

The weighted integrated dominant indices' sums of the alternatives are compared. And the result is shown as follows:

$$
\mathrm{SDI}^{1} \underset{0.5185}{\geq} \mathrm{SDI}^{2} \underset{0.5544}{\geq} \mathrm{SDI}^{3} \underset{0.6144}{\geq} \mathrm{SDI}^{5} \underset{0.6538}{\geq} \mathrm{SDI}^{4} \text {. }
$$

According to the above result, the ranking order of the alternatives is shown below:

$$
a_{1} \underset{0.5185}{\succ} a_{2} \underset{0.5544}{\succ} a_{3} \underset{0.6144}{\succ} a_{5} \underset{0.6538}{\succ} a_{4} .
$$

As a result, the decision maker can select $a_{1}$ as the optimal alternative.

In order to show the superiority of the proposed method, a different method is introduced as follows. In the method, the linguistic evaluation term set is first replaced with $\{1,2,3,4,5,6,7\}$. Then, the optimal probability vector of the states is obtained by the maximum entropy principle [53]. Subsequently, the expected evaluation value of each alternative is gotten under each criterion. Consequently, the interval of the weighted expected evaluation value is computed by the operation laws of the interval numbers for each alternative. The above interval for each alternative is denoted by $\mathrm{WEVI}_{i}(i \in M)$. Thus, the possibility degree matrix and the ranking weight vector of these intervals are calculated. Finally, the ranking order of the alternatives comes out.

Given the limited space available, much of the decision procedure is not to delve into the details.

WEVI $_{i}(i \in M)$ is shown in Table 31.

The possibility degree matrix denoted by $\mathrm{PDM}^{\prime}$ and the ranking weight vector denoted by $\mathrm{RV}^{\prime}$ of the intervals are calculated as follows:

$\mathrm{PDM}^{\prime}$

$$
=\left(\begin{array}{lllll}
0.5000 & 0.5100 & 0.5300 & 0.6585 & 0.5297 \\
0.4900 & 0.5000 & 0.5201 & 0.6496 & 0.5198 \\
0.4700 & 0.4799 & 0.5000 & 0.6295 & 0.4997 \\
0.3415 & 0.3504 & 0.3705 & 0.5000 & 0.3697 \\
0.4703 & 0.4802 & 0.5003 & 0.6303 & 0.5000
\end{array}\right),
$$

$$
\mathrm{RV}^{\prime}=(0.2114,0.2090,0.2040,0.1716,0.2041) .
$$

The ranking order of the alternatives is shown below:

$$
a_{1} \underset{0.5100}{\succ} a_{2} \underset{0.5198}{\succ} a_{5} \underset{0.5003}{\succ} a_{3} \underset{0.6295}{\succ} a_{4} \text {. }
$$

\section{Discussion}

Comparing the above two methods in Section 7, the ranking orders have the only difference between $a_{3}$ and $a_{5}$. In the second method, on the one hand, the difference between the values of $a_{3}$ and $a_{5}$ is only 0.0001 in the ranking weight vector. On the other hand, the possibility degree of $a_{5}>a_{3}$ is only 0.5003 in the possibility degree matrix. All this shows that the first method has the excellent performances. The key cause that the difference between $a_{3}$ and $a_{5}$ is generated in the methods may be explained as follows. The first method is completely constructed on the basis of the objective laws of the data itself. However, the second method involves the subjective consideration of the decision maker when the linguistic evaluation term set is replaced.

In the illustrative example, the discrete linguistic stochastic variables are the discrete stochastic variables evaluated by the simple normal linguistic term sets. In fact, in the proposed method, the discrete stochastic variables can be evaluated by the 2-tuple fuzzy linguistic representation model, multigranularity linguistic term sets, virtual linguistic term sets and so on.

Interval number is a common kind of the incomplete information in decision making. In the proposed method, the state probabilities and criterion weights are interval numbers. They can be expressed by the other kinds of the incomplete 
TABLE 14: $\mathrm{SDM}_{1}$.

\begin{tabular}{cccccc}
\hline & $a_{1}$ & $a_{2}$ & $a_{3}$ & $a_{4}$ & $a_{5}$ \\
\hline$a_{1}$ & $\phi$ & {$[0.4000,0.7400]$} & {$[0.4700,0.8300]$} & {$[0.3700,0.7200]$} & {$[0.2300,0.4800]$} \\
$a_{2}$ & {$[0.0400,0.1500]$} & $\phi$ & {$[0.2000,0.3500]$} & {$[0.2800,0.5300]$} & {$[0.0800,0.2200]$} \\
$a_{3}$ & {$[0.0400,0.1500]$} & {$[0.1600,0.3000]$} & $\phi$ & {$[0.3000,0.5600]$} & {$[0.0900,0.2400]$} \\
$a_{4}$ & {$[0.1500,0.3200]$} & {$[0.2400,0.4400]$} & {$[0.2700,0.4800]$} & $\phi$ & {$[0.2100,0.4000]$} \\
$a_{5}$ & {$[0.2100,0.5400]$} & {$[0.3600,0.6900]$} & {$[0.4300,0.7800]$} & {$[0.4100,0.7500]$} & $\phi$ \\
\hline
\end{tabular}

TABLE 15: $\mathrm{SDM}_{2}$.

\begin{tabular}{cccccc}
\hline & $a_{1}$ & $a_{2}$ & $a_{3}$ & $a_{4}$ & $a_{5}$ \\
\hline$a_{1}$ & $\phi$ & {$[0.1600,0.3300]$} & {$[0.1800,0.3600]$} & {$[0.2400,0.4500]$} & {$[0.2200,0.4200]$} \\
$a_{2}$ & {$[0.4000,0.6900]$} & $\phi$ & {$[0.3000,0.5200]$} & {$[0.4100,0.6600]$} & {$[0.3700,0.6100]$} \\
$a_{3}$ & {$[0.2800,0.5300]$} & {$[0.1800,0.3600]$} & $\phi$ & {$[0.2900,0.5100]$} & {$[0.2600,0.4700]$} \\
$a_{4}$ & {$[0.1600,0.3700]$} & {$[0.1000,0.2500]$} & {$[0.1200,0.2800]$} & $\phi$ & {$[0.1500,0.3300]$} \\
$a_{5}$ & {$[0.2000,0.5300]$} & {$[0.1200,0.3600]$} & {$[0.1500,0.4000]$} & {$[0.2200,0.5100]$} & $\phi$ \\
\hline
\end{tabular}

TABLE 16: $\mathrm{SDM}_{3}$.

\begin{tabular}{cccccc}
\hline & $a_{1}$ & $a_{2}$ & $a_{3}$ & $a_{4}$ & $a_{5}$ \\
\hline$a_{1}$ & $\phi$ & {$[0.5600,0.9900]$} & {$[0.4500,0.7200]$} & {$[0.5600,1.0800]$} & {$[0.3600,0.6000]$} \\
$a_{2}$ & {$[0.1200,0.2400]$} & $\phi$ & {$[0.4000,0.6600]$} & {$[0.4800,0.9600]$} & {$[0.3200,0.5500]$} \\
$a_{3}$ & {$[0.1300,0.3300]$} & {$[0.2400,0.5500]$} & $\phi$ & {$[0.2400,0.6000]$} & {$[0.1400,0.3100]$} \\
$a_{4}$ & {$[0,0]$} & {$[0,0]$} & {$[0.4000,0.7200]$} & $\phi$ & {$[0.3200,0.6000]$} \\
$a_{5}$ & {$[0.2200,0.4500]$} & {$[0.3200,0.6600]$} & {$[0.4400,0.7500]$} & {$[0.3200,0.7200]$} & $\phi$ \\
\hline
\end{tabular}

TABLE 17: $\mathrm{SDM}_{4}$.

\begin{tabular}{cccccc}
\hline & $a_{1}$ & $a_{2}$ & $a_{3}$ & $a_{4}$ & $a_{5}$ \\
\hline$a_{1}$ & $\phi$ & {$[0.2200,0.4500]$} & {$[0.1400,0.2700]$} & {$[0.4000,0.7300]$} & {$[0.2700,0.5200]$} \\
$a_{2}$ & {$[0.3300,0.5600]$} & $\phi$ & {$[0.1500,0.2400]$} & {$[0.4200,0.7300]$} & {$[0.3800,0.6200]$} \\
$a_{3}$ & {$[0.3900,0.8000]$} & {$[0.2600,0.7000]$} & $\phi$ & {$[0.4400,0.9300]$} & {$[0.4600,0.9700]$} \\
$a_{4}$ & {$[0.1800,0.3600]$} & {$[0.1000,0.2400]$} & {$[0.0600,0.1200]$} & $\phi$ & {$[0.2000,0.3900]$} \\
$a_{5}$ & {$[0.2400,0.4400]$} & {$[0.1400,0.3000]$} & {$[0.0600,0.1200]$} & {$[0.4000,0.6900]$} & $\phi$ \\
\hline
\end{tabular}

TABLE 18: $\mathrm{SDM}_{5}$.

\begin{tabular}{cccccc}
\hline & $a_{1}$ & $a_{2}$ & $a_{3}$ & $a_{4}$ & $a_{5}$ \\
\hline$a_{1}$ & $\phi$ & {$[0.2200,0.4200]$} & {$[0.2000,0.4300]$} & {$[0.2900,0.5400]$} & {$[0.4800,0.8800]$} \\
$a_{2}$ & {$[0.2100,0.5400]$} & $\phi$ & {$[0.2000,0.5100]$} & {$[0.3700,0.8100]$} & {$[0.4000,0.8800]$} \\
$a_{3}$ & {$[0.2200,0.4200]$} & {$[0.2600,0.4700]$} & $\phi$ & {$[0.3400,0.6100]$} & {$[0.4800,0.8800]$} \\
$a_{4}$ & {$[0.1000,0.2100]$} & {$[0.1500,0.2800]$} & {$[0.1800,0.3600]$} & $\phi$ & {$[0.4000,0.7700]$} \\
$a_{5}$ & {$[0.1600,0.3300]$} & {$[0,0]$} & {$[0.1600,0.3300]$} & {$[0.3200,0.5500]$} & $\phi$ \\
\hline
\end{tabular}

TABLE 19: IDM $_{1}$.

\begin{tabular}{cccccc}
\hline & $a_{1}$ & $a_{2}$ & $a_{3}$ & $a_{4}$ & $a_{5}$ \\
\hline$a_{1}$ & $\phi$ & {$[0.0400,0.1500]$} & {$[0.0400,0.1500]$} & {$[0.1500,0.3200]$} & {$[0.2100,0.5400]$} \\
$a_{2}$ & {$[0.4000,0.7400]$} & $\phi$ & {$[0.1600,0.3000]$} & {$[0.2400,0.4400]$} & {$[0.3600,0.6900]$} \\
$a_{3}$ & {$[0.4700,0.8300]$} & {$[0.2000,0.3500]$} & $\phi$ & {$[0.2700,0.4800]$} & {$[0.4300,0.7800]$} \\
$a_{4}$ & {$[0.3700,0.7200]$} & {$[0.2800,0.5300]$} & {$[0.3000,0.5600]$} & $\phi$ & {$[0.4100,0.7500]$} \\
$a_{5}$ & {$[0.2300,0.4800]$} & {$[0.0800,0.2200]$} & {$[0.0900,0.2400]$} & {$[0.2100,0.4000]$} & $\phi$ \\
\hline
\end{tabular}


TABLE 20: IDM $_{2}$.

\begin{tabular}{cccccc}
\hline & $a_{1}$ & $a_{2}$ & $a_{3}$ & $a_{4}$ & $a_{5}$ \\
\hline$a_{1}$ & $\phi$ & {$[0.4000,0.6900]$} & {$[0.2800,0.5300]$} & {$[0.1600,0.3700]$} & {$[0.2000,0.5300]$} \\
$a_{2}$ & {$[0.1600,0.3300]$} & $\phi$ & {$[0.1800,0.3600]$} & {$[0.1000,0.2500]$} & {$[0.1200,0.3600]$} \\
$a_{3}$ & {$[0.1800,0.3600]$} & {$[0.3000,0.5200]$} & $\phi$ & {$[0.1200,0.2800]$} & {$[0.1500,0.4000]$} \\
$a_{4}$ & {$[0.2400,0.4500]$} & {$[0.4100,0.6600]$} & {$[0.2900,0.5100]$} & $\phi$ & {$[0.2200,0.5100]$} \\
$a_{5}$ & {$[0.2200,0.4200]$} & {$[0.3700,0.6100]$} & {$[0.2600,0.4700]$} & {$[0.1500,0.3300]$} & $\phi$ \\
\hline
\end{tabular}

TABLE 21: $\mathrm{IDM}_{3}$.

\begin{tabular}{cccccc}
\hline & $a_{1}$ & $a_{2}$ & $a_{3}$ & $a_{4}$ & $a_{5}$ \\
\hline$a_{1}$ & $\phi$ & {$[0.1200,0.2400]$} & {$[0.1300,0.3300]$} & {$[0,0]$} & {$[0.2200,0.4500]$} \\
$a_{2}$ & {$[0.5600,0.9900]$} & $\phi$ & {$[0.2400,0.5500]$} & {$[0,0]$} & {$[0.3200,0.6600]$} \\
$a_{3}$ & {$[0.4500,0.7200]$} & {$[0.4000,0.6600]$} & $\phi$ & {$[0.4000,0.7200]$} & {$[0.4400,0.7500]$} \\
$a_{4}$ & {$[0.5600,1.0800]$} & {$[0.4800,0.9600]$} & {$[0.2400,0.6000]$} & $\phi$ & {$[0.3200,0.7200]$} \\
$a_{5}$ & {$[0.3600,0.6000]$} & {$[0.3200,0.5500]$} & {$[0.1400,0.3100]$} & {$[0.3200,0.6000]$} & $\phi$ \\
\hline
\end{tabular}

TABLE 22: $\mathrm{IDM}_{4}$.

\begin{tabular}{cccccc}
\hline & $a_{1}$ & $a_{2}$ & $a_{3}$ & $a_{4}$ & $a_{5}$ \\
\hline$a_{1}$ & $\phi$ & {$[0.3300,0.5600]$} & {$[0.3900,0.8000]$} & {$[0.1800,0.3600]$} & {$[0.2400,0.4400]$} \\
$a_{2}$ & {$[0.2200,0.4500]$} & $\phi$ & {$[0.2600,0.7000]$} & {$[0.1000,0.2400]$} & {$[0.1400,0.3000]$} \\
$a_{3}$ & {$[0.1400,0.2700]$} & {$[0.1500,0.2400]$} & $\phi$ & {$[0.0600,0.1200]$} & {$[0.0600,0.1200]$} \\
$a_{4}$ & {$[0.4000,0.7300]$} & {$[0.4200,0.7300]$} & {$[0.4400,0.9300]$} & $\phi$ & {$[0.4000,0.6900]$} \\
$a_{5}$ & {$[0.2700,0.5200]$} & {$[0.3800,0.6200]$} & {$[0.4600,0.9700]$} & {$[0.2000,0.3900]$} & $\phi$ \\
\hline
\end{tabular}

TABLE 23: IDM .

\begin{tabular}{cccccr}
\hline & $a_{1}$ & $a_{2}$ & $a_{3}$ & $a_{4}$ & $a_{5}$ \\
\hline$a_{1}$ & $\phi$ & {$[0.2100,0.5400]$} & {$[0.2200,0.4200]$} & {$[0.1000,0.2100]$} & {$[0.1600,0.3300]$} \\
$a_{2}$ & {$[0.2200,0.4200]$} & $\phi$ & {$[0.2600,0.4700]$} & {$[0.1500,0.2800]$} & {$[0,0]$} \\
$a_{3}$ & {$[0.2000,0.4300]$} & {$[0.2000,0.5100]$} & $\phi$ & {$[0.1800,0.3600]$} & {$[0.1600,0.3300]$} \\
$a_{4}$ & {$[0.2900,0.5400]$} & {$[0.3700,0.8100]$} & {$[0.3400,0.6100]$} & $\phi$ & {$[0.3200,0.5500]$} \\
$a_{5}$ & {$[0.4800,0.8800]$} & {$[0.4000,0.8800]$} & {$[0.4800,0.8800]$} & {$[0.4000,0.7700]$} & $\phi$ \\
\hline
\end{tabular}

TABLE 24: $\mathrm{DDM}_{1}$.

\begin{tabular}{cccccc}
\hline & $a_{1}$ & $a_{2}$ & $a_{3}$ & $a_{4}$ & $a_{5}$ \\
\hline$a_{1}$ & $\phi$ & {$[0.2500,0.7000]$} & {$[0.3200,0.7900]$} & {$[0.0500,0.5700]$} & {$[-0.3100,0.2700]$} \\
$a_{2}$ & {$[-0.7000,-0.2500]$} & $\phi$ & {$[-0.1000,0.1900]$} & {$[-0.1600,0.2900]$} & {$[-0.6100,-0.1400]$} \\
$a_{3}$ & {$[-0.7900,-0.3200]$} & {$[-0.1900,0.1000]$} & $\phi$ & {$[-0.1800,0.2900]$} & {$[-0.6900,-0.1900]$} \\
$a_{4}$ & {$[-0.5700,-0.0500]$} & {$[-0.2900,0.1600]$} & {$[-0.2900,0.1800]$} & $\phi$ & {$[-0.5400,-0.0100]$} \\
$a_{5}$ & {$[-0.2700,0.3100]$} & {$[0.1400,0.6100]$} & {$[0.1900,0.6900]$} & {$[0.0100,0.5400]$} & $\phi$ \\
\hline
\end{tabular}

TABLE 25: $\mathrm{DDM}_{2}$.

\begin{tabular}{cccccc}
\hline & $a_{1}$ & $a_{2}$ & $a_{3}$ & $a_{4}$ & $a_{5}$ \\
\hline$a_{1}$ & $\phi$ & {$[-0.5300,-0.0700]$} & {$[-0.3500,0.0800]$} & {$[-0.1300,0.2900]$} & {$[-0.3100,0.2200]$} \\
$a_{2}$ & {$[0.0700,0.5300]$} & $\phi$ & {$[-0.0600,0.3400]$} & {$[0.1600,0.5600]$} & {$[0.0100,0.4900]$} \\
$a_{3}$ & {$[-0.0800,0.3500]$} & {$[-0.3400,0.0600]$} & $\phi$ & {$[0.0100,0.3900]$} & {$[-0.1400,0.3200]$} \\
$a_{4}$ & {$[-0.2900,0.1300]$} & {$[-0.5600,-0.1600]$} & {$[-0.3900,-0.0100]$} & $\phi$ & {$[-0.3600,0.1100]$} \\
$a_{5}$ & {$[-0.2200,0.3100]$} & {$[-0.4900,-0.0100]$} & {$[-0.3200,0.1400]$} & {$[-0.1100,0.3600]$} & $\phi$ \\
\hline
\end{tabular}


TABLE 26: $\mathrm{DDM}_{3}$.

\begin{tabular}{cccccc}
\hline & $a_{1}$ & $a_{2}$ & $a_{3}$ & $a_{4}$ & $a_{5}$ \\
\hline$a_{1}$ & $\phi$ & {$[0.3200,0.8700]$} & {$[0.1200,0.5900]$} & {$[0.5600,1.0800]$} & {$[-0.0900,0.3800]$} \\
$a_{2}$ & {$[-0.8700,-0.3200]$} & $\phi$ & {$[-0.1500,0.4200]$} & {$[0.4800,0.9600]$} & {$[-0.3400,0.2300]$} \\
$a_{3}$ & {$[-0.5900,-0.1200]$} & {$[-0.4200,0.1500]$} & $\phi$ & {$[-0.4800,0.2000]$} & {$[-0.6100,-0.1300]$} \\
$a_{4}$ & {$[-1.0800,-0.5600]$} & {$[-0.9600,-0.4800]$} & {$[-0.2000,0.4800]$} & $\phi$ & {$[-0.4000,0.2800]$} \\
$a_{5}$ & {$[-0.3800,0.0900]$} & {$[-0.2300,0.3400]$} & {$[0.1300,0.6100]$} & {$[-0.2800,0.4000]$} & $\phi$ \\
\hline
\end{tabular}

TABLE 27: $\mathrm{DDM}_{4}$.

\begin{tabular}{cccccc}
\hline & $a_{1}$ & $a_{2}$ & $a_{3}$ & $a_{4}$ & $a_{5}$ \\
\hline$a_{1}$ & $\phi$ & {$[-0.3400,0.1200]$} & {$[-0.6600,-0.1200]$} & {$[0.0400,0.5500]$} & {$[-0.1700,0.2800]$} \\
$a_{2}$ & {$[-0.1200,0.3400]$} & $\phi$ & {$[-0.5500,-0.0200]$} & {$[0.1800,0.6300]$} & {$[0.0800,0.4800]$} \\
$a_{3}$ & {$[0.1200,0.6600]$} & {$[0.0200,0.5500]$} & $\phi$ & {$[0.3200,0.8700]$} & {$[0.3400,0.9100]$} \\
$a_{4}$ & {$[-0.5500,-0.0400]$} & {$[-0.6300,-0.1800]$} & {$[-0.8700,-0.3200]$} & $\phi$ & {$[-0.4900,-0.0100]$} \\
$a_{5}$ & {$[-0.2800,0.1700]$} & {$[-0.4800,-0.0800]$} & {$[-0.9100,-0.3400]$} & {$[0.0100,0.4900]$} & $\phi$ \\
\hline
\end{tabular}

TABLE 28: $\mathrm{DDM}_{5}$.

\begin{tabular}{cccccc}
\hline & $a_{1}$ & $a_{2}$ & $a_{3}$ & $a_{4}$ & $a_{5}$ \\
\hline$a_{1}$ & $\phi$ & {$[-0.3200,0.2100]$} & {$[-0.2200,0.2100]$} & {$[0.0800,0.4400]$} & {$[0.1500,0.7200]$} \\
$a_{2}$ & {$[-0.2100,0.3200]$} & $\phi$ & {$[-0.2700,0.2500]$} & {$[0.0900,0.6600]$} & {$[0.4000,0.8800]$} \\
$a_{3}$ & {$[-0.2100,0.2200]$} & {$[-0.2500,0.2700]$} & $\phi$ & {$[-0.0200,0.4300]$} & {$[0.1500,0.7200]$} \\
$a_{4}$ & {$[-0.4400,-0.0800]$} & {$[-0.6600,-0.0900]$} & {$[-0.4300,0.0200]$} & $\phi$ & {$[-0.1500,0.4500]$} \\
$a_{5}$ & {$[-0.7200,-0.1500]$} & {$[-0.8800,-0.4000]$} & {$[-0.7200,-0.1500]$} & {$[-0.4500,0.1500]$} & $\phi$ \\
\hline
\end{tabular}

TABLE 29: $\mathrm{DDM}_{\omega}$.

\begin{tabular}{cccccc}
\hline & $a_{1}$ & $a_{2}$ & $a_{3}$ & $a_{4}$ & $a_{5}$ \\
\hline$a_{1}$ & $\phi$ & {$[-0.2580,0.4245]$} & {$[-0.2600,0.3845]$} & {$[0.0490,0.7150]$} & {$[-0.2010,0.4955]$} \\
$a_{2}$ & {$[-0.4245,0.2580]$} & $\phi$ & {$[-0.2915,0.3055]$} & {$[0.0930,0.7880]$} & {$[-0.1260,0.5490]$} \\
$a_{3}$ & {$[-0.3845,0.2600]$} & {$[-0.3055,0.2915]$} & $\phi$ & {$[-0.0965,0.5760]$} & {$[-0.2555,0.4885]$} \\
$a_{4}$ & {$[-0.7150,-0.0490]$} & {$[-0.7880,-0.0930]$} & {$[-0.5760,0.0965]$} & $\phi$ & {$[-0.4905,0.2205]$} \\
$a_{5}$ & {$[-0.4955,0.2010]$} & {$[-0.5490,0.1260]$} & {$[-0.4885,0.2555]$} & {$[-0.2205,0.4905]$} & $\phi$ \\
\hline
\end{tabular}

TABLE 30: $\operatorname{SDI}^{i_{1}}\left(i_{1} \in M=\{1,2, \ldots, 5\}\right)$.

\begin{tabular}{lc}
\hline$a_{i_{1}}$ & $\mathrm{SDI}^{i_{1}}$ \\
\hline$a_{1}$ & {$[-0.6700,2.0195]$} \\
$a_{2}$ & {$[-0.7490,1.9005]$} \\
$a_{3}$ & {$[-1.0420,1.6160]$} \\
$a_{4}$ & {$[-2.5695,0.1750]$} \\
$a_{5}$ & {$[-1.7535,1.0730]$} \\
\hline
\end{tabular}

information or the missing values. It is a direction for further research on the proposed method.

The state space can be divided into the consistent one and the inconsistent one. In the consistent state space, the numbers of the states of the discrete stochastic variables are the same. In the inconsistent state space, the numbers of the states of the discrete stochastic variables are not the same. In the proposed method, the consistent state space is considered. The method is flexible, and it can be extended to the inconsistent state space.
TABle 31: $\mathrm{WEVI}_{i}(i \in M)$.

\begin{tabular}{lc}
\hline$a_{i}(i \in M)$ & $\mathrm{WEVI}_{i}(i \in M)$ \\
\hline$a_{1}$ & {$[4.4725,6.4925]$} \\
$a_{2}$ & {$[4.4550,6.4300]$} \\
$a_{3}$ & {$[4.3925,6.3350]$} \\
$a_{4}$ & {$[4.0200,5.7550]$} \\
$a_{5}$ & {$[4.4000,6.3300]$} \\
\hline
\end{tabular}

Linguistic stochastic information reveals well the subjective fuzziness and objective randomness of the complex uncertain phenomenon. In the actual decision making problems, there exist a lot of such phenomena in the catastrophic emergency management, financial risk monitoring and logistics management. These are concrete potential applications of the proposed method.

To specific issues in the LSMCDM problems, obtaining the accurate decision making data, constructing the scientific 
decision making model, and making the optimal decision are worthy of widespread and in-depth research in future.

\section{Acknowledgments}

This work is supported by the Natural Science Foundation of Hunan Province in China (no. 10JJ6107), the Philosophy and Social Science Foundation of Hunan Province in China (nos. 09YBA072, 11YBA232, and 12YBA195), the Scientific Research Foundation of Education Department of Hunan Province in China (no. 10C0792), the National Natural Science Foundation of China (nos. 71071163 and 61273232), the National Social Science Foundation (no. 12BJY055), and the Social Science Research Foundation of the Ministry Of Education (nos. 12YJAZH142 and 11YJA870024).

\section{References}

[1] L. A. Zadeh, "The concept of a linguistic variable and its application to approximate reasoning-I," Information Sciences, vol. 8, no. 3, pp. 199-249, 1975.

[2] L. A. Zadeh, "The concept of a linguistic variable and its application to approximate reasoning-II," Information Sciences, vol. 8, no. 4, pp. 301-357, 1975.

[3] L. A. Zadeh, "The concept of a linguistic variable and its application to approximate reasoning-III," Information Sciences, vol. 9, no. 1, pp. 43-80, 1975.

[4] F. Herrera and L. Martinez, "A 2-tuple fuzzy linguistic representation model based on a symbolic translation," in Proceedings of the EUROFUSE-SIC'99 Conference, pp. 25-28, Budapest, Hungary, 1999.

[5] F. Herrera and L. Martinez, "An approach for combining linguistic and numerical information based on the 2-tuple fuzzy linguistic representation model in decision-making," International Journal of Uncertainty, Fuzziness and KnowlegeBased Systems, vol. 8, no. 5, pp. 539-562, 2000.

[6] J. H. Wang and J. Y. Hao, "A new version of 2-tuple fuzzy linguistic representation model for computing with words," IEEE Transactions on Fuzzy Systems, vol. 14, no. 3, pp. 435-445, 2006.

[7] F. Herrera, E. Herrera-Viedma, and L. Martínez, "A fuzzy linguistic methodology to deal with unbalanced linguistic term sets," IEEE Transactions on Fuzzy Systems, vol. 16, no. 2, pp. 354370, 2008.

[8] Y. Dong, Y. Xu, and S. Yu, "Computing the numerical scale of the linguistic term set for the 2-tuple fuzzy linguistic representation model," IEEE Transactions on Fuzzy Systems, vol. 17, no. 6, pp. 1366-1378, 2009.

[9] Y. C. Dong, W. C. Hong, Y. F. Xu, and S. Yu, "Selecting the individual numerical scale and prioritization method in the analytic hierarchy process: a 2-tuple fuzzy linguistic approach," IEEE Transactions on Fuzzy Systems, vol. 19, no. 1, pp. 13-25, 2011.

[10] L. Martínez and F. Herrera, "An overview on the 2-tuple linguistic model for computing with words in decision making: extensions, applications and challenges," Information Sciences, vol. 207, pp. 1-18, 2012.

[11] F. Herrera, E. Herrera-Viedma, and L. Martínez, "A fusion approach for managing multi-granularity linguistic term sets in decision making," Fuzzy Sets and Systems, vol. 114, no. 1, pp. 43$58,2000$.
[12] Z. S. Xu, "An approach to pure linguistic multiple attribute decision making under uncertainty," International Journal of Information Technology and Decision Making, vol. 4, no. 2, pp. 197-206, 2005.

[13] Z. S. Xu, "A method based on linguistic aggregation operators for group decision making with linguistic preference relations," Information Sciences, vol. 166, no. 1-4, pp. 19-30, 2004.

[14] Y. P. Jiang, Z. P. Fan, and J. Ma, "A method for group decision making with multi-granularity linguistic assessment information," Information Sciences, vol. 178, no. 4, pp. 1098-1109, 2008.

[15] W. P. Wang, "Toward developing agility evaluation of mass customization systems using 2-tuple linguistic computing," Expert Systems with Applications, vol. 36, no. 2, pp. 3439-3447, 2009.

[16] Y. C. Dong, Y. F. Xu, and S. Yu, "Linguistic multiperson decision making based on the use of multiple preference relations," Fuzzy Sets and Systems, vol. 160, no. 5, pp. 603-623, 2009.

[17] Z. S. Xu, "An interactive approach to multiple attribute group decision making with multigranular uncertain linguistic information," Group Decision and Negotiation, vol. 18, no. 2, pp. 119145, 2009.

[18] C. M. Liu, M. J. Wang, and Y. S. Pang, "A multiple criteria linguistic decision model (MCLDM) for human decision making," European Journal of Operational Research, vol. 76, no. 3, pp. 466-485, 1994.

[19] Z. S. Xu, "Deviation measures of linguistic preference relations in group decision making," Omega, vol. 33, no. 3, pp. 249-254, 2005.

[20] Z. S. Xu, "An interactive procedure for linguistic multiple attribute decision making with incomplete weight information," Fuzzy Optimization and Decision Making, vol. 6, no. 1, pp. 17-27, 2007.

[21] D. Ben-Arieh, "Sensitivity of multi-criteria decision making to linguistic quantifiers and aggregation means," Computers and Industrial Engineering, vol. 48, no. 2, pp. 289-309, 2005.

[22] Y. J. Xu and Q. L. Da, "A method for multiple attribute decision making with incomplete weight information under uncertain linguistic environment," Knowledge-Based Systems, vol. 21, no. 8, pp. 837-841, 2008.

[23] F. Chiclana, E. Herrera-Viedma, and S. Alonso, "A note on two methods for estimating missing pairwise preference values," IEEE Transactions on Systems, Man, and Cybernetics B, vol. 39, no. 6, pp. 1628-1633, 2009.

[24] J. M. Mendel, "Historical reflections and new positions on perceptual computing," Fuzzy Optimization and Decision Making, vol. 8, no. 4, pp. 325-335, 2009.

[25] J. M. Mendel and D. Wu, Perceptual Computing: Aiding People in Making Subjective Judgments, IEEE Press and John Wiley \& Sons, Hoboken, NJ, USA, 2010.

[26] B. Mareschal, "Stochastic multicriteria decision making and uncertainty," European Journal of Operational Research, vol. 26, no. 1, pp. 58-64, 1986.

[27] J. M. Martel and K. Zaras, "Stochastic dominance in multicriterion analysis under risk," Theory and Decision, vol. 39, no. 1, pp. 31-49, 1995.

[28] M. Nowak, "Preference and veto thresholds in multicriteria analysis based on stochastic dominance," European Journal of Operational Research, vol. 158, no. 2, pp. 339-350, 2004.

[29] M. Nowak, "Interactive approach in multicriteria analysis based on stochastic dominance," Control and Cybernetics, vol. 33, no. 3, pp. 463-476, 2004. 
[30] M. Nowak, "INSDECM-an interactive procedure for stochastic multicriteria decision problems," European Journal of Operational Research, vol. 175, no. 3, pp. 1413-1430, 2006.

[31] Y. Zhang, Z. P. Fan, and Y. Liu, "A method based on stochastic dominance degrees for stochastic multiple criteria decision making," Computers and Industrial Engineering, vol. 58, no. 4, pp. 544-552, 2010.

[32] R. Lahdelma, J. Hokkanen, and P. Salminen, "SMAAstochastic multiobjective acceptability analysis," European Journal of Operational Research, vol. 106, no. 1, pp. 137-143, 1998.

[33] R. Lahdelma and P. Salminen, "SMAA-2: stochastic multicriteria acceptability analysis for group decision making," Operations Research, vol. 49, no. 3, pp. 444-454, 2001.

[34] A. Félix, A. Baquerizo, J. M. Santiago, and M. A. Losada, "Coastal zone management with stochastic multi-criteria analysis," Journal of Environmental Management, vol. 112, pp. 252266, 2012.

[35] R. Lahdelma, P. Salminen, and J. Hokkanen, "Locating a waste treatment facility by using stochastic multicriteria acceptability analysis with ordinal criteria," European Journal of Operational Research, vol. 142, no. 2, pp. 345-356, 2002.

[36] A. Menou, A. Benallou, R. Lahdelma, and P. Salminen, "Decision support for centralizing cargo at a Moroccan airport hub using stochastic multicriteria acceptability analysis," European Journal of Operational Research, vol. 204, no. 3, pp. 621-629, 2010.

[37] R. Lahdelma and P. Salminen, "Pseudo-criteria versus linear utility function in stochastic multi-criteria acceptability analysis," European Journal of Operational Research, vol. 141, no. 2, pp. 454-469, 2002.

[38] R. Lahdelma and P. Salminen, "Stochastic multicriteria acceptability analysis using the data envelopment model," European Journal of Operational Research, vol. 170, no. 1, pp. 241-252, 2005.

[39] R. Lahdelma and P. Salminen, "Prospect theory and stochastic multicriteria acceptability analysis (SMAA)," Omega, vol. 37, no. 5, pp. 961-971, 2009.

[40] T. Tervonen, J. R. Figueira, R. Lahdelma, J. A. Dias, and P. Salminen, "A stochastic method for robustness analysis in sorting problems," European Journal of Operational Research, vol. 192, no. 1, pp. 236-242, 2009.

[41] K. Zaras, "Rough approximation of a preference relation by a multi-attribute stochastic dominance for determinist and stochastic evaluation problems," European Journal of Operational Research, vol. 130, no. 2, pp. 305-314, 2001.

[42] K. Zaras, "Rough approximation of a preference relation by a multi-attribute dominance for deterministic, stochastic and fuzzy decision problems," European Journal of Operational Research, vol. 159, no. 1, pp. 196-206, 2004.

[43] Z. P. Fan, Y. Liu, and B. Feng, "A method for stochastic multiple criteria decision making based on pairwise comparisons of alternatives with random evaluations," European Journal of Operational Research, vol. 207, no. 2, pp. 906-915, 2010.

[44] M. Zarghami, F. Szidarovszky, and R. Ardakanian, "A fuzzystochastic OWA model for robust multi-criteria decision making," Fuzzy Optimization and Decision Making, vol. 7, no. 1, pp. $1-15,2008$.

[45] L. Liu, Y. X. Chen, and Z. H. Ge, "Probability measure of interval-number based on normal distribution and multiattribute decision making," Systems Engineering and Electronics, vol. 30, no. 4, pp. 652-654, 2008 (Chinese).
[46] J. Q. Wang and L. Gong, "Interval probability fuzzy random multi-criteria decision-making approach based on expectationhybrid entropy," Control and Decision, vol. 24, no. 7, pp. 10651069, 2009 (Chinese).

[47] J. Ren and Y. Gao, "Discrete fuzzy-stochastic multi-criterion decision-making method with incomplete information," System Engineering Theory and Practice, vol. 31, no. 1, pp. 122-130, 2011 (Chinese).

[48] H. L. Wang and Y. Q. Feng, "On multiple attribute group decision making with linguistic assessment information based on cloud model," Control and Decision, vol. 20, no. 6, pp. 679685, 2005 (Chinese).

[49] J. H. Hu, X. H. Chen, and Y. M. Liu, "Multi-criteria decision making method based on linguistic evaluation and prospect theory," Control and Decision, vol. 24, no. 10, pp. 1477-1482, 2009 (Chinese).

[50] J. Ren, "Linguistic-stochastic multi-criterion decision-making method based on cloud model," Computer Integrated Manufacturing Systems, vol. 18, no. 12, pp. 2792-2797, 2012 (Chinese).

[51] E. Herrera-Viedma, F. Chiclana, F. Herrera, and S. Alonso, "Group decision-making model with incomplete fuzzy preference relations based on additive consistency," IEEE Transactions on Systems, Man, and Cybernetics B, vol. 37, no. 1, pp. 176-189, 2007.

[52] M. W. Wang, "Bayesian decision method based on probability interval," Systems Engineering-Theory and Practice, vol. 17, no. 11, pp. 79-82, 1997 (Chinese).

[53] J. Ren and Y. Gao, "Stochastic multi-criterion decision-making method based on interval operation," Systems Engineering and Electronics, vol. 32, no. 2, pp. 18-22, 2010 (Chinese). 


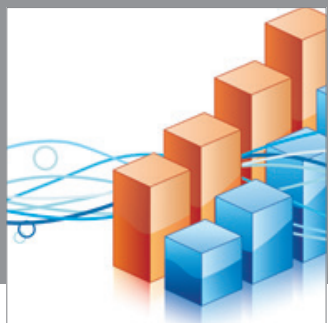

Advances in

Operations Research

mansans

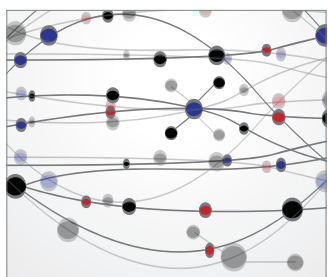

The Scientific World Journal
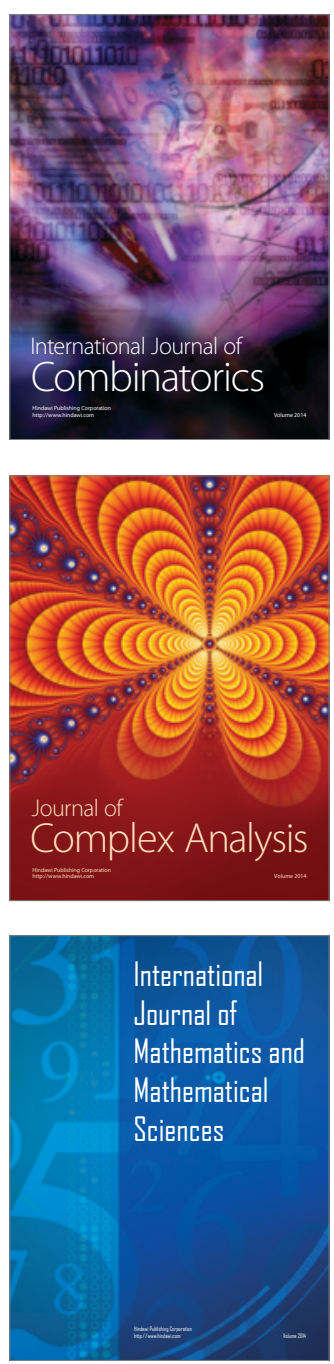
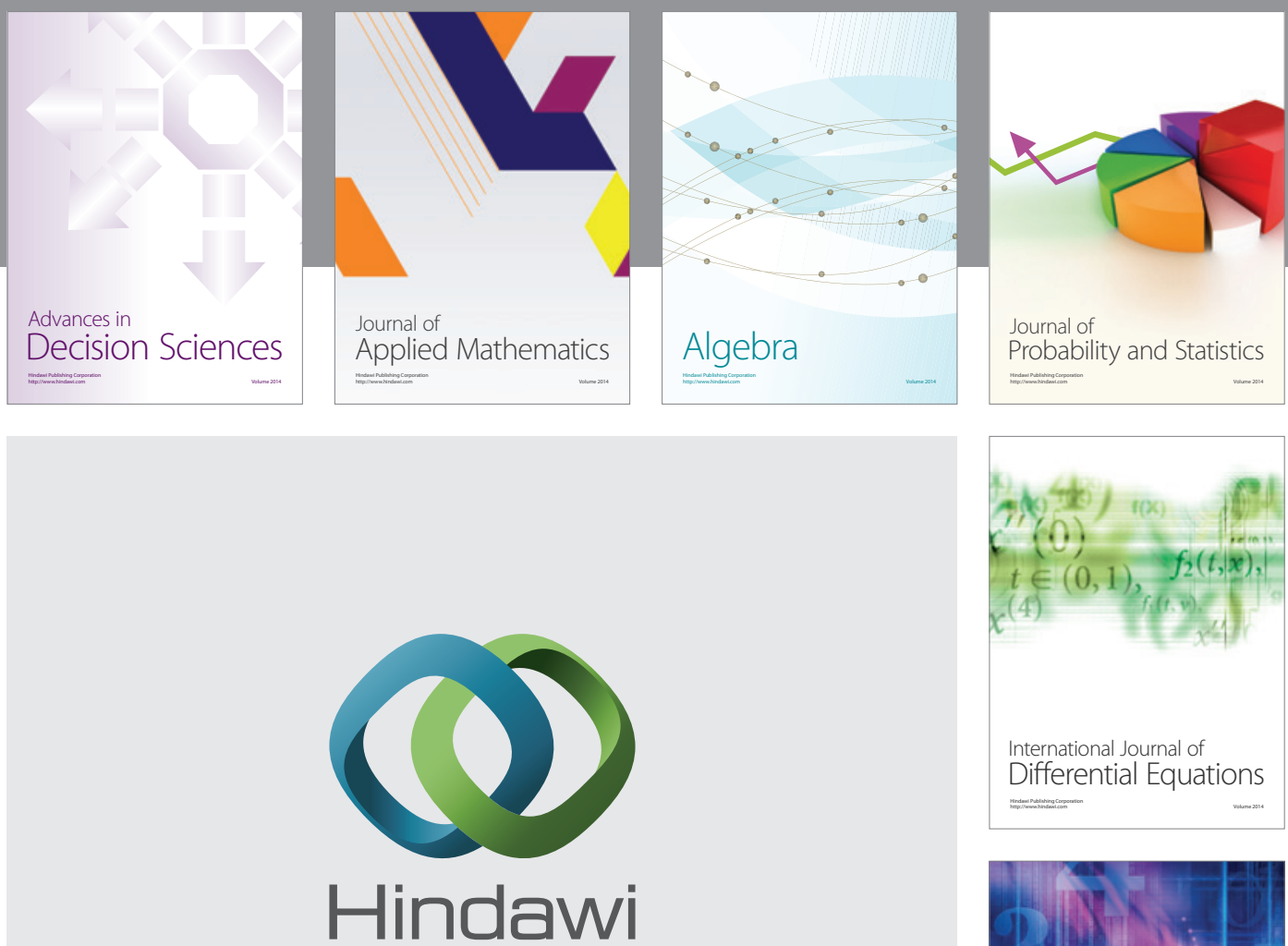

Submit your manuscripts at http://www.hindawi.com
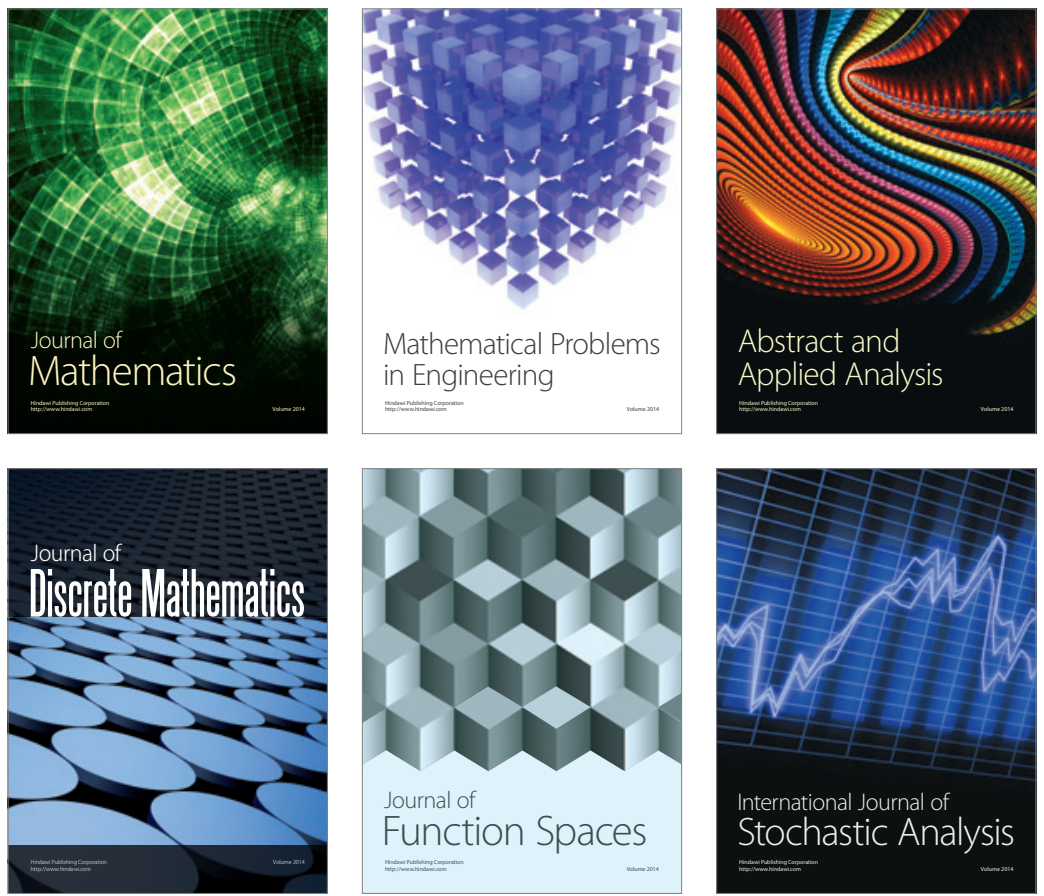

Journal of

Function Spaces

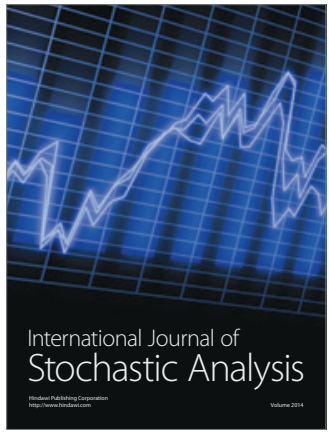

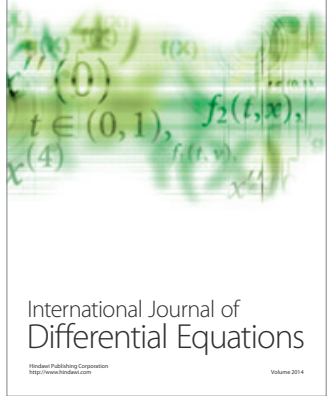
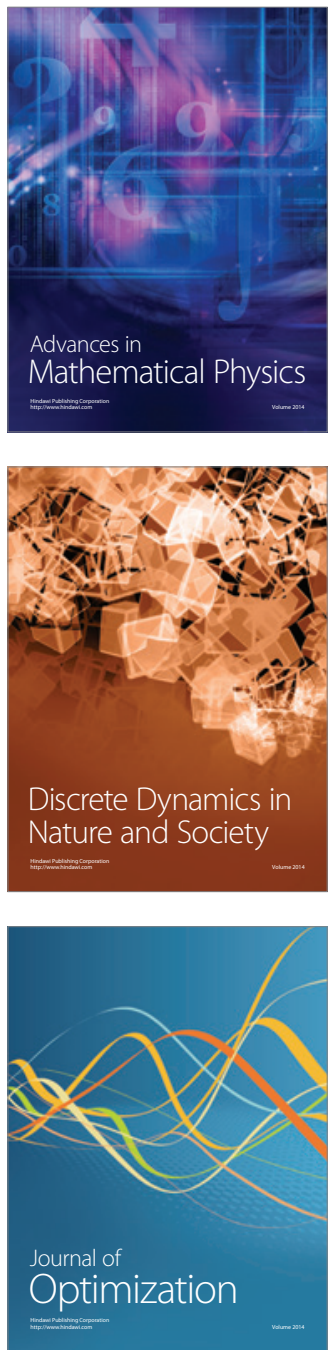